\title{
USING AHP/ANP IN SUPPORT OF MEDICAL DIAGNOSIS
}

\author{
Claudio A. Garuti*, Mario M. Sandoval \\ Fulcrum Ingeniería Ltda. \\ Lu is Thayer Ojeda 0180 Of.1004 - Providencia \\ Santiago - Chile \\ e-mail: claudiogaruti@fulcrum.cl
}

\begin{abstract}
The objective of this presentation, is to show a support tool for decision making in the medical diagnosis area based in the use of AHP/ANP, where the huge numbers of variables and knowledge needed to structure, integrate and synthesize all the information related to illnesses and the patient's health condition requires a solid and systemic approach, able to deal with such complexity. The system is currently under testing mode
\end{abstract}

Keywords: Diagnosis, AHP/ANP, Multidisciplinary, illness, illness profiles, Medical Sapiens.

\section{Introduction:}

The medical diagnosis is a complex problem mainly because is based on signs that have different degree of intensity, evolution and duration, as well as symptoms that depend on the patient's perception. Physicians should interpret all this information and integrate it with the patient's illness history, with lab exams and with their own life and professional experience. Until now, medic ine based on evidence has provided week medical diagnos is, intensifying more than necessary the need of significant clinical studies for each given patient and his specific health conditions, recognizing the patient as a unique and unrepeatable individual.

More experienced physicians are recognized by the ir peers as they have internally developed some sort of integrative information format, whose application over time leads to better and quicker diagnosis (being a good pattern recognitioner). Even if limited by the scope of their direct or relatively direct experience. This soft data (provided by a life of professional experience), has not been captured in most medic ine books yet, but jointly with labs exams and the patient's illness history may be structured in a series of hierarchy models under a network system, that will become the core of a system oriented to provide support to physicians on medical diagnosis and assist to spread knowledge beyond the ir immediate scope of action, for example in the schools of medicine as a tool to teach how to integrate the experience in a formal diagnos is process.

The Medical Sapiens Software (MSS), in counterposition of the classic differential medicine and Bayesian based systems, is oriented to build a diagnosis profile for a specific patient based in an additive (not differential) way of thinking and is not refusing to use the medicine based on evidence. It is founded on the analysis of patient's symptoms and signs in an additive model, without cut any possible diagnoses result until the very last moment, complemented by his/her

\footnotetext{
${ }^{*}$ Corresponding author
} 
health history and selected lab exams, as well as the use of underlying health models developed by physicians with demonstrated expertise in each treated field, that may be improved over time in a learning feed-back process. The main idea of this system is based in two steps:

1.- Using the AHP/ANP models build a diagnose profile of the patient (the anamnesis process) and then,

2.- Measure the compatibility (closeness) between the patient profile with a significant set of recognized disease profiles already knows and stored in a large Data Base, calculating the degree of compatibility (closeness) of each disease profile with the patient, providing a reduced and prioritized set of possible diagnosis that physician finally considers, (gaining also some degree of efficiency by focalizing the physician effort).

An important element of this process is to be able to make an accurate measure of the compatibility between the two profiles (patient and data base profiles). Also, this compatibility or closeness has to be measured in a weighted environment, inside this space of measurement the length in one axe is not equivalent with the length in any other, and the zero value is not always well defined, this condition drive to the necessity of build a new kind of compatibility measure (compatibility index) able to deal with these conditions.

\section{Figure 1: Measuring Compatibility (Closeness) in a Weighted Environment}

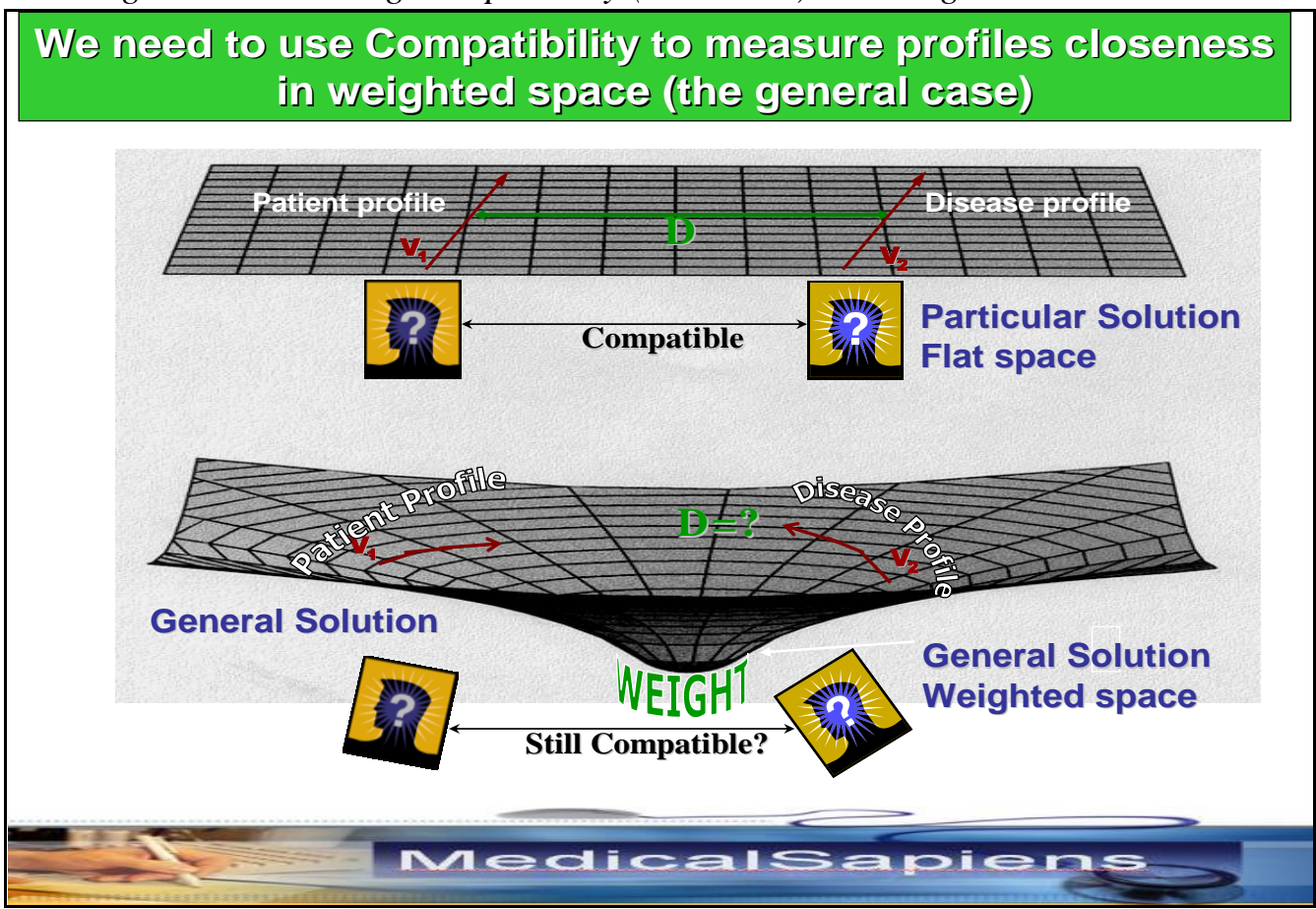

The complementary information like patient's history and lab exams may act as a filter to reduce the initial illnesses list.

With the AHP, we are able to divide a very complex problem in a control hierarchy that have a first level of strategic criteria (symptoms and signs), then expand each one of these criteria into more specific subcriteria until reach the terminal criteria, they are the illness behavior indicators (pain, fever, hematuria, etc..), then build a specific scale for each terminal criterion that have the capacity of measure the behavior of the alternatives (the different diagnosis profiles). 
Later, makes judgments or performs measurements on pairs of elements with respect to a controlling element (using the eigenvector systemic operator), to derive acceptable consistent ratio scales that are then synthesized throughout the structure, then evaluating the set of illness alternatives, and then select the illness profiles that match better with the illness patient profile. The last is done through a new compatibility index $(G)$ built it especially for complex profiles that belongs to weighted environments (compatibility index for weighted profiles). This compatibility index, is able to assess accurately the closeness between the set of illness diagnosis profiles (standard profiles), and the patient illness specific profile. The more close the patient illness is to one illness standard profile the more likely the patient illness became (see figure2).

Figure 2: Compatibility Index between Patient Profile Diagnose and a Set of Disease Patterns Profiles

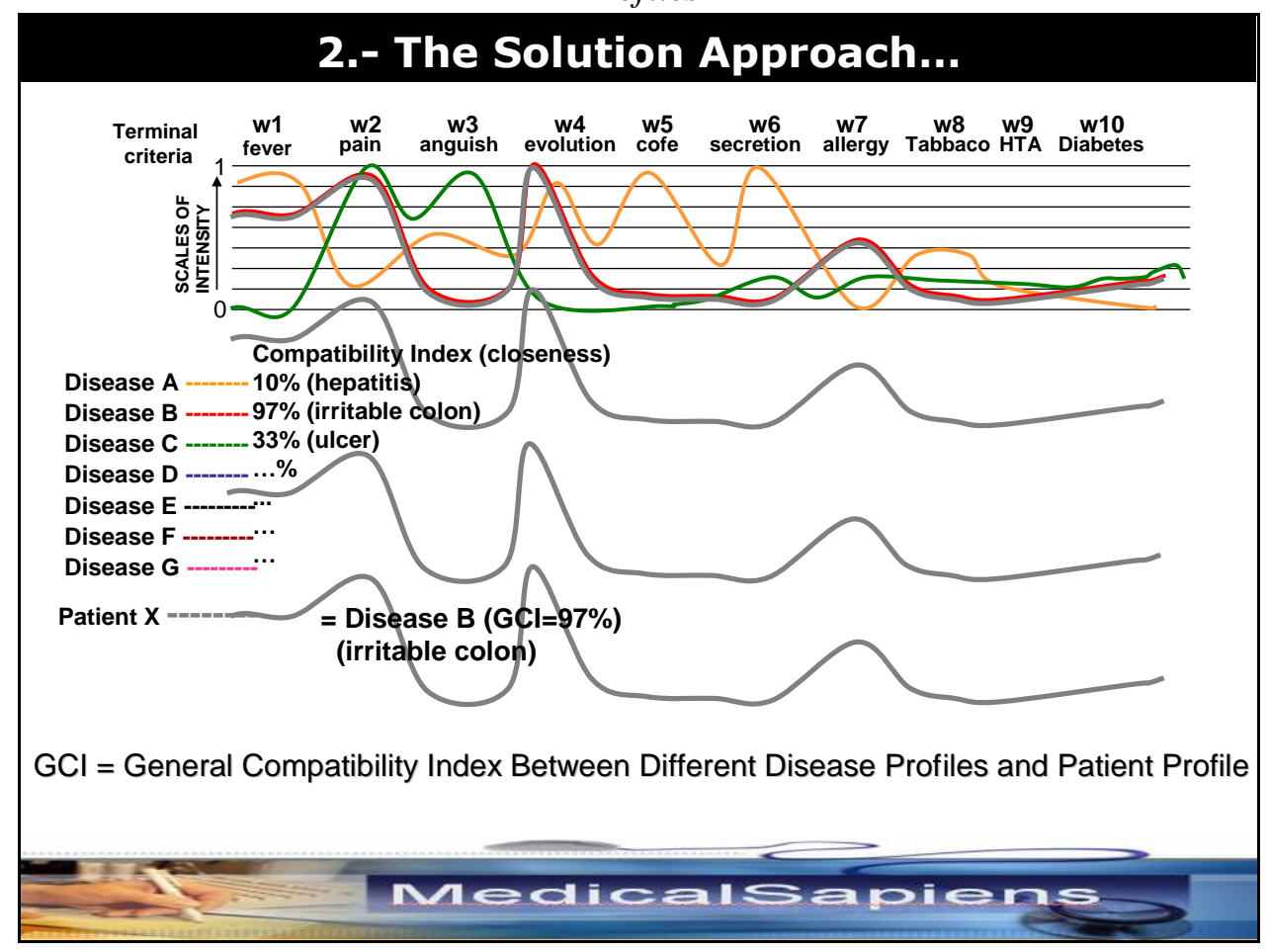

\section{A Short Overview to the Process:}

The physicians with more experience have a great advantage in front of others that have recently ended the ir studies, since they make better and faster integrative information format which lead to a better and quickly diagnosis. This soft data (life experience), haven't been captured in the medicine books but, trough the AHP/ANP we were able to capture this crucial line of information (life experience). This information, jointly with labs exams and patient historic antecedents have been structured in a hierarchy and network model making a system oriented to give a support for medical diagnosis to the physicians.

The system goal is to build a diagnosis profile for a specific patient, founded in the analys is of the symptom and signs of the patient, these signs and symptoms have a specific weight obtained from a pairwise comparison of physicians with demonstrate great expertise in each treated field (a good doctor is a good pattern recognition of diseases). Then, the diagnosis profile is modulated by the antecedents and lab exams. In some occasions, the antecedents and lab exams act as a filter or border condition action, and in some other cases act as a modulator of the weights in the ranking of profile illness. 
The system is also able to provide multiple diagnoses for a patient suffering multiple illnesses, but is not able yet to include the synergy between them; this interaction belongs to the prognosis area which is a delicate physician decis ion making area that has to be explored in detail later.

This diagnosis system software (called Medical Sapiens), is built over the internet platform and includes modules that help the physic ian to collect the patient's data in a structured and readable way (the patient file history), also orienting on what information might be more relevant to be required and followed.

It is important to note the educational possibilities provided by this system, helping medicine students to quickly understand how the more experimented and wisdom doctors make their diagnosis, to what signs and symptoms they should pay more attention under a given situation and orient complementary exams over more focused topics, transferring part of their expertise to the new breed.

The web based system is called Medical Sapiens and it's initial version is based on 33 integrated AHP/ANP models, currently on testing mode.

\section{REFERENCES}

Fundamentals of Decision Making \& Priority Theory with the Analytic Hierarchy Process, Thomas L. Saaty, 527 pp., 1994.

Decision Making with Dependence and Feedback: The Analytic Network Process. T. L. Saaty, 386 pp., 2001.

Theory and Applications of the Analytic Network Process: Decision Making With Benefits, Opportunities, Costs and Risks T. L. Saaty, 335 pp., 2005

A mathematical approach to medical diagnos is: application to congenital heart disease. H.R. Warner, A.F. Toronto, L.G. Veasy, and R. Stephenson

Bayesian Belief Networks: Odds and Ends (1996), Linda C. van der Gaag

Toma De Decisiones En Escenarios Complejos, Garuti C. Escudey M, 2005. Editorial Universidad de Santiago.

When Close Really Mean Close". Garuti C. 2007. International Symposium of AHP (ISAHPIX,2007), Viña del Mar, Chile. 